\title{
Aerial Robot Coworkers for Autonomous Localization of Missing Tools in Manufacturing Plants
}

\author{
J. Ramiro Martínez-de Dios ${ }^{1}$, Arturo Torres-González ${ }^{1}$, Julio L. Paneque ${ }^{1}$, Dimas Fuego-García ${ }^{1}$, \\ Juan Ramón Astorga Ramírez ${ }^{2}$ and Aníbal Ollero ${ }^{1}$
}

\begin{abstract}
This paper presents an aerial robot capable of collaborating with humans for detecting, identifying and locating missing tools in manufacturing and assembly industrial plants. The proposed system has been installed and extensively validated in the Airbus A320 Neo fan-cowl factory in Centro Bahía de Cádiz (CBC) at Puerto de Santa María (Cádiz, Spain). The validation experiments took between December 2017 to February 2018 and the system was partially operated by the AIRBUS DS workers while factory was in full production. To the best of our knowledge this is the very first aerial robotic system cooperating with humans in manufacturing that has been validated in factory production conditions. The paper briefly presents the system, argues on its advantages over traditional approaches and presents experimental results.
\end{abstract}

\section{INTRODUCTION}

There is a strong demand in improving the level of automation in manufacturing industries. Industry 4.0 is a current trend that proposes the increase in the automation and data exchange in manufacturing through the massive introduction of technologies such as cyber-physical systems, the Internet of things, cloud computing and a new generation of robots capable of cooperating with humans in manufacturing, the so-called robot coworkers. Only ground robots have been traditionally employed in factory automation. Aerial robots instead of ground robots have significant advantages in factory automation. First, aerial robots have higher flexibility and speed. Also, factory floors are usually very dense with static and mobile obstacles -e.g. workerswhile the upper part of factories are in most cases completely empty. However, despite these advantages, to the best of our knowledge very few aerial robots for manufacturing have been developed or even experimented.

We are interested in autonomous aerial robots capable of helping workers finding missing tools in factories. Many manufacturing processes require a high number and variety of specialized tools. For instance, more than 100 different tools are necessary for the assembly of every single fan cowl in an aeronautic assembly factory. Missing tools is a severe problem in aeronautic industries due to two main reasons: 1) it may induce significant delays in the production line with evident impact on productivity, and 2) missing items may cause FOD (Foreign Object Damage) if they are forgotten inside an aero-structure. FOD is a critical issue in aeronautic industry with serious impact on product quality.

\footnotetext{
${ }^{1}$ Robotics, Vision and Control Group, University of Seville, Seville, Spain [ jdedios, arturotorres, jlpaneque, aollero] dus.es

${ }_{2}$ Airbus Defence and Space, Centro Bahía de Cádiz, Cádiz, Spain juan.r.astorga@airbus.com
}

Many manufacturing factories are equipped with intelligent tool closets, capable of detecting when a specific tool is missing at the end of the working shift. When a tool is considered missing, it is searched in the factory requiring significant human labor.

This paper presents an aerial robotic system for autonomous detection, identification and location of missing tools in manufacturing plants. In short, each tool is equipped with a radio tag with an ID that can listen and respond to request messages. The aerial robot is equipped with a radio emitter that can send requests and receive responses and take range measurements from missing tools. The range measurements received by the aerial robot as it autonomously navigates in the factory are used to estimate on-board and in real time the location of every missing tool using a combination of Particle Filters (PFs) and Extended Kalman Filters (EKFs). The objective is to estimate the location of each missing tool with enough accuracy so that it can be easily found.

The proposed system has been installed and extensively validated in the Airbus A320 Neo fan-cowl factory in Centro Bahía de Cádiz (CBC) at Puerto de Santa María (Cádiz, Spain) shown in Fig. 1. The validation experiments took between December 2017 to February 2018 and the system was partially operated by the AIRBUS DS workers while factory was in full production.

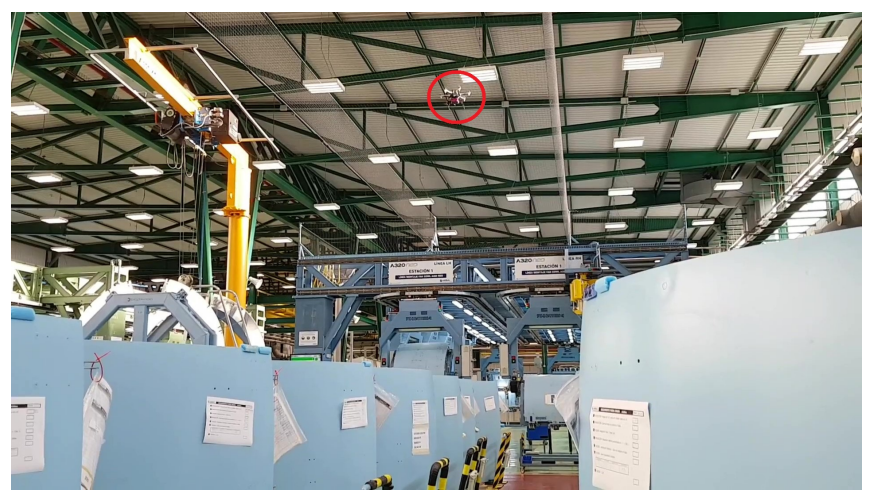

Fig. 1: Picture taken at the Airbus Centro Bahía de Cádiz (CBC) factory at Puerto de Santa María (Cádiz, Spain) during a the operation of the presented system. The aerial robot is marked with a circle.

The presented system has been performed within the framework of project ARCOW (Aerial Robot Co-Worker in Plant Servicing). The main goal of ARCOW is the imple- 
mentation and validation of Micro Aerial Vehicles (MAV) enabling technologies that will allow the introduction of aerial robots collaborating with humans in aircraft manufacturing plants. ARCOW has been developed in the context of European Robotics Challenges EUROC project ${ }^{1}$ funded by the European Commission. ARCOW has been selected in successive qualifying stages as one of the two finalists on EUROC Challenge 3 between more than 35 proposals. In January 2017 ARCOW was awarded with the "Best dronebased solution" in the 'EU Drone Awards'.

This paper is structured as follows. Section II summarizes the main related work in the topics addressed in ARCOW. Section III provides a general description of the presented method arguing the advantages of the aerial robot coworker over other alternatives. The core methods employed for tool search in the presented system are summarized in Section IV including some results from real experiments. Section V describes the development process focusing on the migration from a system tested in the laboratory to the validation in a factory in full production conditions. The conclusions are in the last section.

\section{RELATED WORK}

To the best of our knowledge this is the very first autonomous aerial robotic system cooperating with humans in manufacturing that has been validated in factory full production conditions. Introducing autonomous aerial robot coworkers in manufacturing factories have important advantages in terms of efficiency and cost reduction but also involves significant issues that must be addressed. Below, the state of the art in the main constraining technologies and techniques is briefly summarized.

The robust and safe autonomous robot navigation in complex, obstacle dense, unstructured and changing environments is critical in our problem. Autonomous robot navigation requires estimating the robot position and orientation. This is commonly carried out by computing the robot odometry using an exteroceptive sensor. Some methods combine inertial sensors and altitude sensors with monocular vision systems to estimate the robot localization based on simultaneous localization and mapping (SLAM) [1], [2], [3]. Stereovision systems provide direct depth measurements in a general 3D environment. SLAM and pure odometry approaches demonstrate good results at short-term localization and even in long trajectories without loop closing [4], [5]. Many of the algorithms developed for monocular odometry and SLAM can be applied to stereo cameras based on pattern projection [6], [7], [8]. Some other methods rely on range measurements to sensor nodes deployed in the environment. These methods require performing deployments in the environment but, on the other hand, provide robust measurements, which are very interesting when combined with visual estimations, more sensitive to changes in the scenario and to lighting condition.

\footnotetext{
${ }^{1}$ http://www.euroc-project.eu/
}

Robustness is critical in our problem. Sensor fusion has been pointed out as key to improve robustness in robot localization and navigation [9]. The approach adopted in ARCOW exploits the synergy between different sensors to reduce uncertainty and increase robustness in robot localization and navigation.

There are many robot localization methods based on SLAM, such as RGBD-SLAM [10], ORB-SLAM [6] or RTAB-Map [11]. They build their maps online and do not exploit the fact that the robot moves in a partially known environment. The adopted approach performs robot $6 \mathrm{DoF}$ localization assuming the map is available, which enables reducing SLAM-based robot localization errors.

Introducing autonomous MAVs cooperating with humans in manufacturing in fully factory operational conditions involves challenges from safety and robustness perspectives and requires specific step-by-step procedures to guide human-robot interaction. Recent commercial products show primary positioning abilities and/or collision avoidance capabilities, but are not mature enough to work safely in real and highly dynamic scenarios performing reliably during long periods of time in large industrial environments with strong changes in lighting conditions and strong wind perturbations due to proximity to air conditioning openings, which may have relevant impact on the small aerial vehicles. For example, the DJI Guidance Safety Guidelines include a number of usage notices that restrict their operation depending on lighting conditions, the surroundings structures or presence of moving objects.

In our system tools are detected, identified and located using radio signals. The main methods for radio-based localization are summarized below. Most localization methods with radio tags use the radio signal strength (RSS) as a range measurement. However, reflections and other interactions with the environment, such as multi-path propagation, make RSS measurements very dependent on the scenario. Other technologies can be used to estimate the distance between two radio tags, such as time of flight (TOF). TOF sensors are more accurate than RSS.

Localization with range-only measurements has the problem of partial observability: only one measurement is insufficient to constrain the target location. Thus, the robot has to move and integrate measurements from different positions in order to initialize the locations of the targets. Different approaches have been developed to solve this problem. Multilateration [12], [13] is one of the simplest methods to determine the target position. The robot gathers measurements of the target from different locations, and then tries to estimate its pose through a simple least squares optimization. Multilateration is computationally efficient, but lacks robustness: it is very sensible to measurement noise and outliers, and it can cause bad initialization, which can lead to significant estimation errors. WCL (Weighted Centroid Localization) exhibits high robustness against noise in the RSSI measurements. [14] demonstrated that although other methods, like for example Least Squares (LS), are optimal with noiseless RSSI measurements, the performance of WCL 
is better with realistic (non-Gaussian) levels of noise.

Probability grids [15], [16] provide more robustness than multilateration but are not scalable to large scenarios and their accuracy is related to the size of the cells in the grid. Particle Filters (PFs) [17], [18] can represent any probability distribution. In this case the probability distribution has the shape of a ring in $2 \mathrm{D}$ or an sphere in $3 \mathrm{D}$. As more range measurements are integrated, the particles tend to converge to the real distribution. PFs can be computationally hard, but they can provide a pure probabilistic solution to the partial observability problem. Kalman Filters are very useful for location estimation for mono-hypothesis problems and hence cannot be directly applied in our problem.

Our system employs a combination of PFs and Extended Kalman Filters. PFs are used used to solve for initializing tool localization, i.e. reducing to one the number of potential tool location hypothesis. After initialization an EKF is used to refine the location estimation.

\section{AERIAL ROBOTS FOR TOOL SEARCH}

The new manufacturing paradigm in the aerospace sector pursues the acceleration of delivery rates through the gradual implementation of automated processes. Aerial robots can contribute to this automation process by providing intrinsic capabilities, such as flexibility, fast response and availability. Airbus DS is very interested in the introduction of automation in the Airbus future factory strategy.

Automatic tool search in factories can be addressed using different technologies and approaches. For instance, a system of static sensor nodes could be installed in the factory in order to continuously localize all the missing tools. Nevertheless, this would require an ad hoc installation for each factory, so it would not be suitable for dynamic factories, where the structure and conditions of the factory has frequent changes. Another approach could be installing the sensor nodes on each of the worker's clothes (the helmet would perform properly in this task due to the height where it is located). However, this is not a very feasible approach for this problem as it would compromise the workers' safety and comfort.

In our system, tool detection and search is performed by an aerial robot. When a tool is considered missing, its ID is inserted into the list of the tools to be searched. During the next regular autonomous flights of the aerial robot, e.g. for performing logistic operations, the aerial robot transmits request messages with the IDs of the list of missing tools. When the radio tag of a missing tool receives a request with its ID, it responds. The aerial robot receives the responses together with range measurements and then it estimates the location of the missing tool in the factory map using the techniques summarized in Section IV. The system can search many missing tools simultaneously. Also, it is able to perform missing tool search flights on demand. For example, at the end of a working shift the IDs of the missing tools are inserted in the list and a search flight is performed on demand.
Figure 2 shows the 3D screen provided to workers after the tool search mission showing the resulting locations of three missing tools. That experiment was performed in February 22nd 2018. The estimated locations of the missing tools are shown as light green ellipses. Blue spheres represent the real location of the tools. The factory working areas are represented in light gray. For safety the robot flight zone in the factory was restricted. The boundaries of the flight zones are shown in dark gray in the picture. The trajectories performed by the aerial robot in this tool search mission are shown in red.

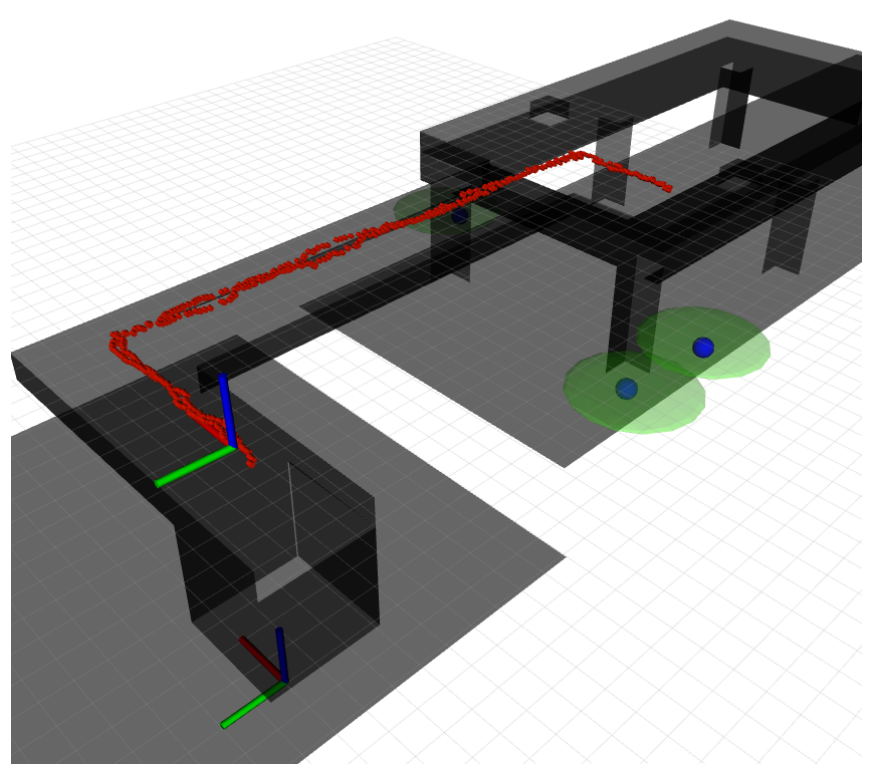

Fig. 2: 3D view with the resulting locations of three missing tools provided by the proposed system in one experiment performed in February 22nd 2018.

Besides the functionalities for autonomous navigation, the aerial robot implements the communication protocol with missing tools and algorithms for estimating their location. Of course, performance in everyday operational conditions in the factory imposes strong safety and robustness requirements.

\section{TECHNIQUES}

The aerial robot is capable of detecting and locating the tools using only its pose and the range measurements it receives from the missing radio-tagged tools. The proposed method uses Particle Filters (PFs) to estimate an initial location of the tool and then, refines this estimation with an EKF. The PFs solve the initial multi-hypothesis problem inherent to range-only measurements whereas the EKFs improve the tool location estimation more efficiently.

The proposed method employs one PF-EKF for every missing tool. Range measurements from each tool are associated with different radio-tag ID, naturally solving the measurement association problem. Assuming that a high number of tools $(N)$ are missing, it is more computationally efficient to have $N$ simpler EKFs than the same number 
of more complex PFs. Thus, the combination of these two different filters allows to solve the multi-hypothesis problem and then to efficiently maintain a good estimation. Another advantage is that individual PFs have faster convergence than more complex multi-tool PFs, involving significantly lower computational resources. The aerial robot performs tool search while doing other tasks, such as logistics, and many other programs are running simultaneously on-board the aerial robot.

When the aerial robot receives the first range measurement $z_{i}$ from tool $i$, it initializes a PF, $P F_{i}$, in which the particles, which are hypotheses of the tool location, are spread around the aerial robot at distances drawn from an annular distribution with mean $z_{i}$ and a width that depends on the measurements variance. From this time on, every time the aerial robot receives a new measurement from tool $i$, the particles of $P F_{i}$ are updated making them to condensate towards the tool's real position. This step estimates the importance factor or weight of each particle, i.e. the probability of a certain particle to represent the real state. Thus, higher weights represent more probable samples. The likelihood function used for estimating the importance factor is:

$$
p^{[j]}(x)=\frac{1}{\sigma \sqrt{2 \pi}} e^{\frac{\left(\left|x[j]-x_{r}\right|-z_{i}\right)^{2}}{2 \sigma^{2}}},
$$

where $p^{[j]}(x)$ is the probability of particle $j$ of representing the true state of $x . x^{[j]}$ is the position of particle $j . x_{r}$ is the aerial robot location. $z_{i}$ is the current measurement of tool $i$. $\sigma$ is the variance of the measurement noise.

PFs also implement the so-called importance resampling, which draws with replacement of $M$ particles. The probability of drawing each particle is given by its importance factor. This step helps to have a faster convergence of the $\mathrm{PF}$, removing particles with low probabilities and duplicating those with higher probabilities. In our case $M=10 \%$ of the particles were replaced.

In our system we considered that a PF has converged in a Gaussian distribution when the maximum eigenvalue of the covariance matrix is lower than a certain threshold. The maximum eigenvalue is a measure of the volume of the confidence ellipsoid of the distribution. Using the eigenvalues establishes the convergence condition on each axis. When $P F_{i}$, the PF for tool $i$, converges, the tool estimated location $x_{i}$ is computed as the weighted mean of all particles, see Eq. (2), and it is used to initialize $E K F_{i}$, the EKF for tool $i$. The flexibility of PFs is at the expense of increasing computer burden. PF iterations require significantly more computer burden than EKF iterations. When the particles converge to a Gaussian-like distribution, the estimation of the missing tool $i$ will be continuously updated with $E K F_{i}$.

$$
x_{i}=\sum_{j=1}^{M} \omega_{i}^{[j]} x_{i}^{[j]}
$$

Missing tools are supposed to be static. Thus, the EKF prediction model will be also static: $x_{t}=x_{t-1}$. On the other hand, the observation model will be the euclidean distance between the aerial robot and the missing tool:

$$
z_{i}=\left|x_{i}-x_{r}\right|
$$

$E K F_{i}$ will refine the estimation obtained with the $P F_{i}$ consuming less computation resources. More details of the algorithm can be found in [19] and [20].

Figure 3 shows several steps in the execution of the PF-EKF method for tool search and localization including: (a) particle deployment; (b-c) several steps in the PF convergence; (d) start of the EKF stage; (e) evolution of the EKF estimation and; (f) final tool location estimation. That experiment is the same as that in Fig. 2. The aerial robot take-off and landing location is shown at the bottom-right corner of the images. The path is represented by red points. The particles of a PF are deployed when the first measurement from that radio tag is received, Fig. 3-a. The method requires integrating several range measurements gathered as the robot navigates. After enough measurements have been integrated, the PFs converge and the EKF location estimates are represented with ellipses. When the robot lands the tools' estimated location computed by the EKFs are provided to the workers in a geometrical 3D representation, see Fig. 3f. Particle aggregations far from the ellipses represent low weighted particles that will be resampled in future steps.

\section{FROM THE LABORATORY TO THE FACTORY}

Our goal is to provide the plant personnel with a fully autonomous system to help them in their daily activities to accelerate delivery rates and reduce lead times and recurring cost. This involves:

- Safe interaction with human workers. This involves the design of an operational procedure which should maintain the safety of the workers but also the ability to interact with them taking into account the usual working conditions in the factory.

- Gradual introduction in the aircraft manufacturing processes taking into account a realistic practical approach. It should include a very safe first implementation with current reliable conventional drones with enough payload to solve a large number of logistic processes. An elevated net has been used in the first safe implementation. Intrinsically safe MAVs with enough payload will be used also in future implementations.

A pragmatic approach taking into account the constraint of the industrial process has been adopted. This strategy allows to accelerate the transition from TRL 4 (technology validated in lab) towards TRLs 5 and 6 (technology validated and demonstrated in industrially relevant environments) which has been reached in February 2018.

The first step is the extensive testing of the methods in laboratory where a wide variety of conditions and experiments were considered, see Fig. 4-top. This stage enabled increasing the robustness of the algorithms. Second, the proposed system has been extensively refined and validated using a realistic mock-up shown in Fig. 4-center. This stage 


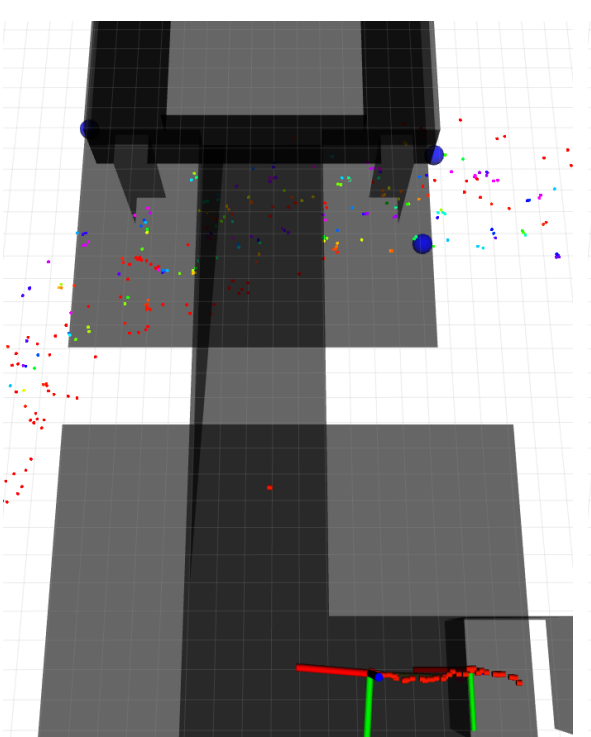

(a)

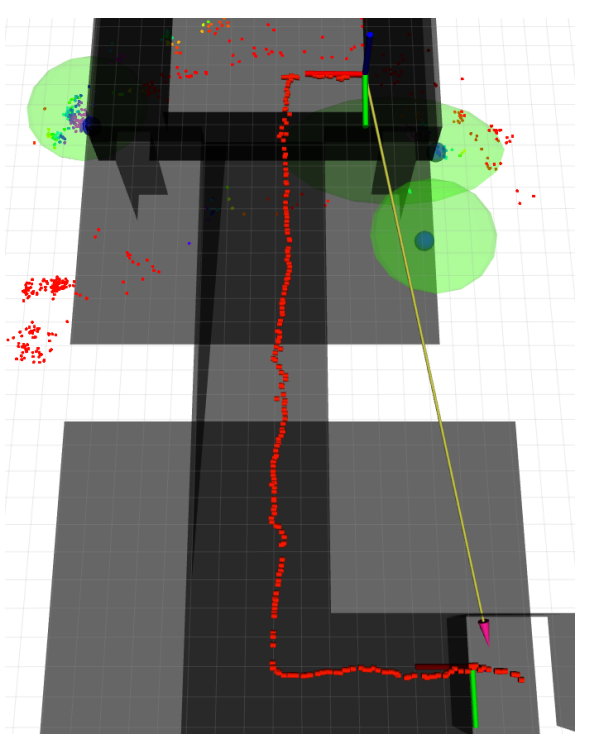

(d)

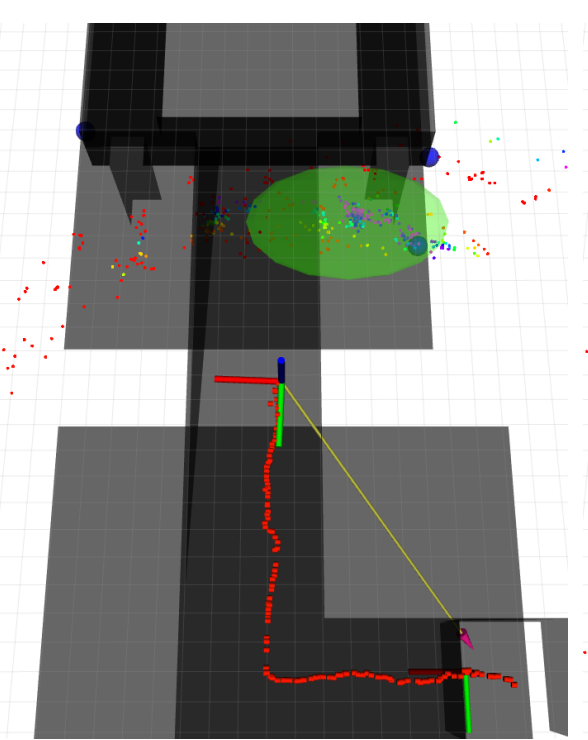

(b)

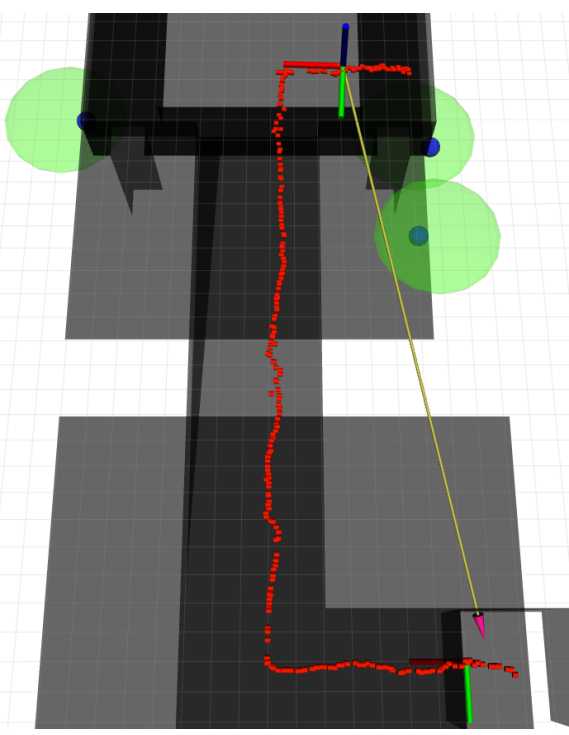

(e)

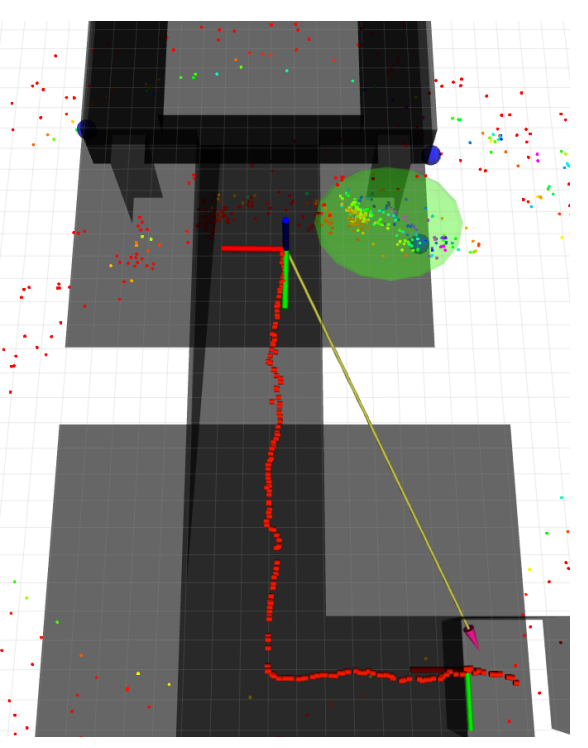

(c)

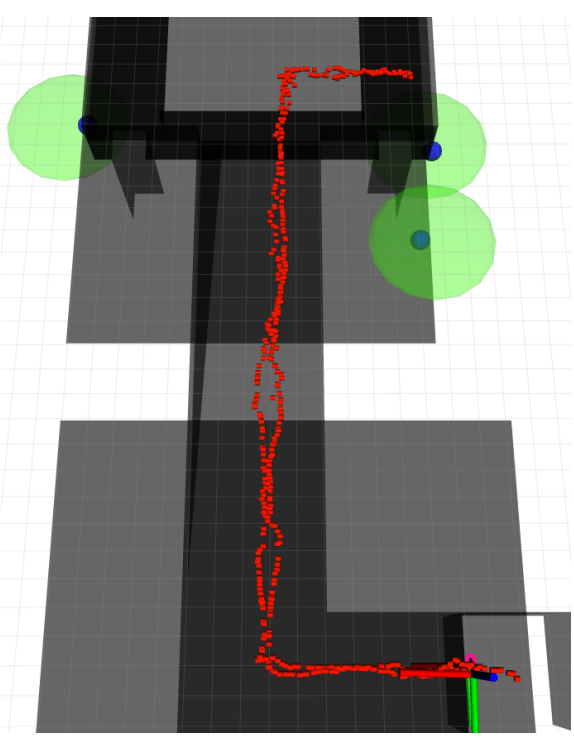

(f)

Fig. 3: Several steps in the execution of the PF-EKF method for tool search and localization of 3 tools: (a) PF particle deployment; (b-c) several steps in the PF convergence; (d) start of the EKF stage; (e) evolution of the EKF estimation and; (f) final tool location estimation.

enabled increasing the performance of the system in realistic conditions.

Finally, the system has been installed and validated in fully working conditions in the Airbus CBC factory at Cadiz (Spain), see Figure 4-bottom. Validating in a real factory imposes many constraints of various types: it was approached in three main steps. From September to November, the system experiments were performed during evenings and nights in order to avoid interactions with the production process. The objective was to adapt the system to the real scenario. In December and January, the experiments took place in the afternoon shift, while the factory was with low-working load. Some Airbus workers were involved in the experiments. The objective was to refine the operational performance of the system and integration with Airbus production systems in partial working conditions. In February 2018, the system was validated during the morning shift in a with full-working load factory and with close interaction of Airbus workers.

In the validation experiments in the real factory, the algorithm was able to correctly locate $95 \%$ of missing tools. The mean accuracy achieved in the experiments was 30 $\mathrm{cm}$. The accuracy in the factory depends highly on the surroundings of the tool's location. For instance, if the tool is hided under several metallic structures, a lot of measurements will be lost and the accuracy will decrease (up to 1 meter).

The robustness of the methods employed is also an issue. Factories have high density of metallic structures. To cope with that, we adopted the real-time radio-range modeling 

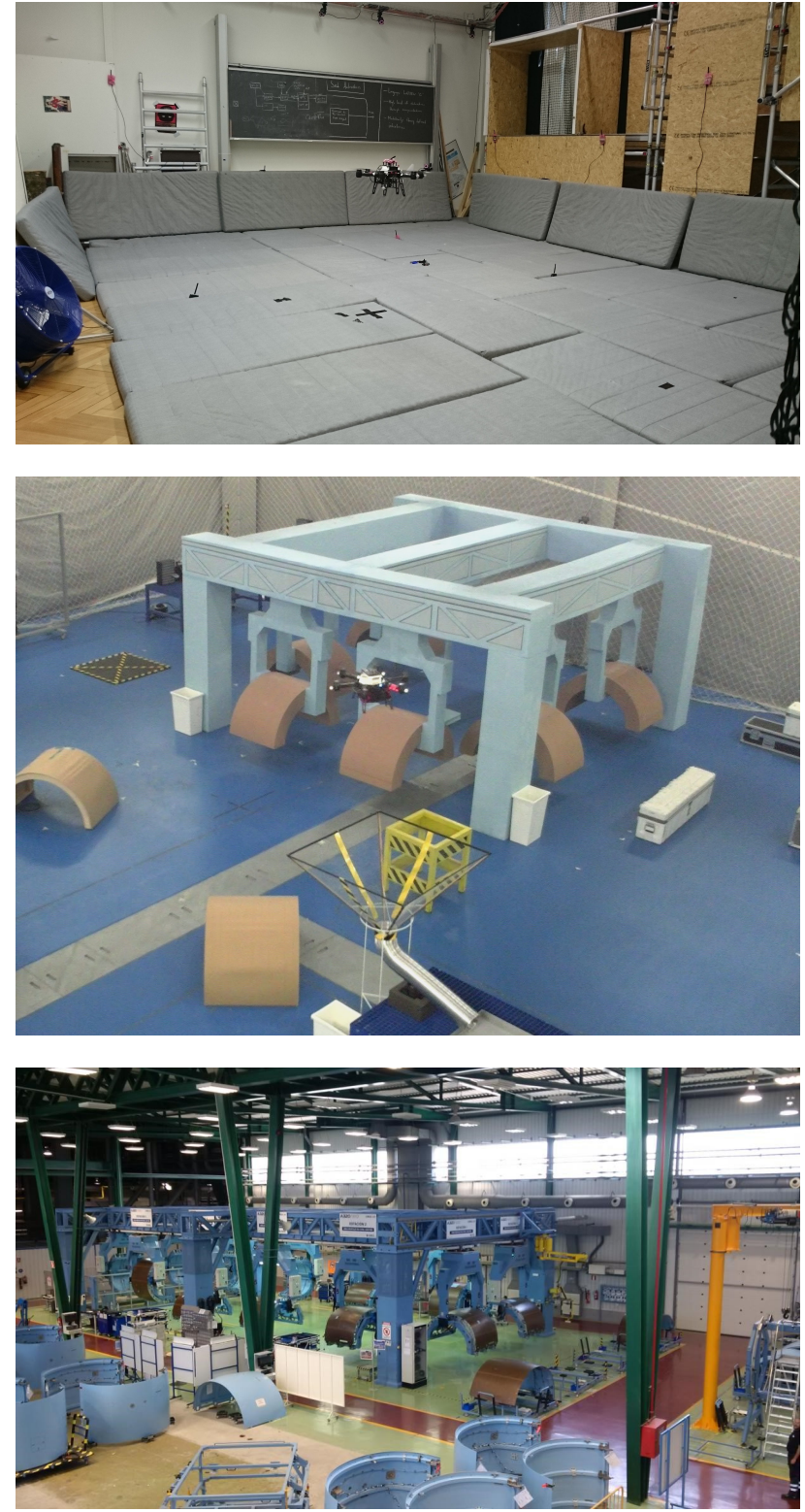

Fig. 4: Pictures of the system development in three main stages: testing in the lab (top); validation in realistic environments with industry plant mock-ups (center) and; validation in the Airbus CBC factory at Cadiz (Spain) (bottom).

technique previously developed by the authors [21] that calibrated the UWB sensors. The system should also be secure from an information perspective. Also, a Public Key Infrastructure (PKI) software architecture involving the aerial robots and the sensor nodes has been developed to provide secure communication and operation.

\section{CONCLUSIONS}

There is a strong interest in improving the level of automation in manufacturing industries by the introduction of robots helping and collaborating with humans. Although traditionally only ground robots have been employed in factory automation, aerial robots have higher flexibility and use the upper part of factories, which are most cases completely empty.

This paper presents an aerial robotic system for autonomous detection, identification and location of missing tools in manufacturing plants. Many manufacturing processes require a high number and variety of specialized tools. Missing tools is a severe problem in aeronautic industries. Missing tool search is time consuming and often results in production delays. In the proposed system a radio tag is attached to each tool and the aerial robot is equipped with a radio emitter that takes range measurements to the missing tools. As the aerial robot navigates autonomously in the factory it integrates on-board, in real-time, the measurements and estimates the location of every missing tool using a combination of Particle Filters (PFs) and Extended Kalman Filters (EKFs).

The proposed system has been extensively validated between December 2017 to February 2018 in the Airbus A320 Neo fan-cowl factory in Centro Bahía de Cádiz (CBC) at the Spanish province of Cádiz while factory was in full production.

This system has been implemented after more than three years of work in the ARCOW project performing tasks such as: the design and testing of the algorithms in the laboratory; the refinement in realistic environments and; the validation in the real factory. Our first objective in the exploitation plan is to actually demonstrate the use of this technology and also validate that the factories are able to obtain the estimated savings. CBC Airbus DS factory in Cádiz will be used as the pilot program and also as the "first client" of this technology. Furthermore, it will be a great marketing advertisement due to the position and brand of Airbus DS in Europe, and in the world. Finally, it is important to highlight that it could also help to create a place for this technology in aircraft manufacturing operations in general.

\section{ACKNOWLEDGMENT}

This work has been performed within the context of EuRoC-ARCOW project funded by the European Commission under contract 608849. Partial funding has been also obtained from UE-funded project AEROARMS project (contract H2020-ICT-2014-1-644271). The authors would like to thank Antidio Viguria, Francisco Javier Pérez-Grau and José Joaquín Acevedo from CATEC.

\section{REFERENCES}

[1] K. Kapach and Y. Edan, "Evaluation of grid-map sensor fusion mapping algorithms," in Conference Proceedings - IEEE International Conference on Systems, Man and Cybernetics, 2007, pp. 829-834.

[2] M. Guo, D. Li, G. Chen, Y. Xu, W. He, T. Zhang, L. An, and M. Lv, "Multi-sensor information fusion for unmanned cars using radar map," in Proceedings - 2012 IEEE 2nd International Conference on Cloud Computing and Intelligence Systems, IEEE CCIS 2012, vol. 3, 2013, pp. 1166-1170.

[3] M. Nagai, T. Chen, R. Shibasaki, H. Kumagai, and A. Ahmed, "UAV-borne 3-D mapping system by multisensor integration," IEEE Transactions on Geoscience and Remote Sensing, vol. 47, no. 3, pp. 701-708, 2009 
[4] C. Forster, Z. Zhang, M. Gassner, M. Werlberger, and D. Scaramuzza, "SVO: Semidirect Visual Odometry for Monocular and Multicamera Systems," IEEE Transactions on Robotics, vol. 33, no. 2, pp. 249-265, 2017.

[5] J. Engel, V. Koltun, and D. Cremers, "Direct sparse odometry," IEEE Transactions on Pattern Analysis and Machine Intelligence, Mar. 2018.

[6] R. Mur-Artal, J. M. Montiel, and J. D. Tardos, "ORB-SLAM: A Versatile and Accurate Monocular SLAM System," IEEE Transactions on Robotics, vol. 31, no. 5, pp. 1147-1163, 2015.

[7] J. Engel, T. Schöps, and D. Cremers, "LSD-SLAM: Large-Scale Direct monocular SLAM," in Lecture Notes in Computer Science (including subseries Lecture Notes in Artificial Intelligence and Lecture Notes in Bioinformatics), vol. 8690 LNCS, no. PART 2, 2014, pp. 834-849.

[8] R. A. Newcombe, S. J. Lovegrove, and A. J. Davison, "DTAM: Dense tracking and mapping in real-time," in Proceedings of the IEEE International Conference on Computer Vision, 2011, pp. 2320-2327.

[9] S. Lynen, M. W. Achtelik, S. Weiss, M. Chli, and R. Siegwart, "A robust and modular multi-sensor fusion approach applied to MAV navigation," in IEEE International Conference on Intelligent Robots and Systems, 2013, pp. 3923-3929.

[10] Y. Bergeon, I. Hadda, V. Kivánek, J. Motsch, and A. Štefek, "Low cost 3D mapping for indoor navigation," International Conference on Military Technologies (ICMT), 2015.

[11] M. Labbe and F. Michaud, "Appearance-based loop closure detection for online large-scale and long-term operation," IEEE Transactions on Robotics, vol. 29, no. 3, pp. 734-745, 2013.

[12] X. Wang, O. Bischoff, R. Laur, and S. Paul, "Localization in wireless ad-hoc sensor networks using multilateration with rssi for logistic applications," Procedia Chemistry, vol. 1, no. 1, pp. 461-464, 2009.

[13] A. Wessels, X. Wang, R. Laur, and W. Lang, "Dynamic indoor localization using multilateration with rssi in wireless sensor networks for transport logistics," Procedia Engineering, vol. 5, pp. 220-223, 2010.

[14] J. Blumenthal, R. Grossmann, F. Golatowski, and D. Timmermann, "Weighted centroid localization in zigbee-based sensor networks," in Intelligent Signal Processing, 2007. WISP 2007. IEEE International Symposium on. IEEE, 2007, pp. 1-6.

[15] R. Stoleru and J. A. Stankovic, "Probability grid: A location estimation scheme for wireless sensor networks," in Sensor and Ad Hoc Communications and Networks, 2004. IEEE SECON 2004. 2004 First Annual IEEE Communications Society Conference on. IEEE, 2004, pp. 430-438.

[16] G. Kantor and S. Singh, "Preliminary results in range-only localization and mapping," in Robotics and Automation, 2002. Proceedings. ICRA'02. IEEE International Conference on, vol. 2. Ieee, 2002, pp. $1818-1823$.

[17] S. Thrun, D. Fox, W. Burgard, and F. Dellaert, "Robust monte carlo localization for mobile robots," Artificial intelligence, vol. 128, no. 1-2, pp. 99-141, 2001.

[18] M. K. Pitt and N. Shephard, "Filtering via simulation: Auxiliary particle filters," Journal of the American statistical association, vol. 94, no. 446 , pp. 590-599, 1999.

[19] A. Torres-González, J. R. Martinez-de Dios, and A. Ollero, "Rangeonly slam for robot-sensor network cooperation," Autonomous Robots, pp. 1-15, 2017.

[20] J. L. Paneque, A. Torres-González, J. R. Martínez-de Dios, J. R. A. Ramírez, and A. Ollero, "Autonomous localization of missing items with aerial robots in an aircraft factory," in Iberian Robotics conference. Springer, Cham, 2017, pp. 179-189.

[21] J. R. Martnez-de Dios, A. Ollero, F. J. Fernndez, and C. Regoli, "Online rssi-range model learning for target localization and tracking," Journal of Sensor and Actuator Networks, vol. 6, no. 3, 2017. 